\title{
Elliptic Self-Similar Stochastic Processes
}

\author{
Albert Benassi and Daniel Roux
}

\begin{abstract}
Let $M$ be a random measure and $L$ be an elliptic pseudo-differential operator on $\mathbb{R}^{d}$. We study the solution of the stochastic problem $L X=M, X(0)=0$ when some homogeneity and integrability conditions are assumed. If $M$ is a Gaussian measure the process $X$ belongs to the class of Elliptic Gaussian Processes which has already been studied. Here the law of $M$ is not necessarily Gaussian. We characterize the solutions $X$ which are self-similar and with stationary increments in terms of the driving measure $M$. Then we use appropriate wavelet bases to expand these solutions and we give regularity results. In the last section it is shown how a percolation forest can help with constructing a self-similar Elliptic Process with non stable law.
\end{abstract}

\section{Introduction}

\subsection{Self-similarity, ellipticity}

The scale invariance property plays a fundamental role in many areas of theoretical and applied sciences. A single word can unify different occurrences of this concept: the word fractal, as coined by Mandelbrot in [9]. Roughly speaking a fractal set $F$ presents the same aspect at different scales of observation: every little part of $F$ looks like the entire object after zooming. A mathematical definition of this property is "self-similarity".

The class of self-similar stochastic processes indexed by $\mathbb{R}^{d}$ is very large, even if we only consider processes with stationary increments as we shall do in the sequel. A complete description is known in the Gaussian case since the results of Dobrushin [5], but this is not longer true in the general case, even when restricted to stable laws.

2000 Mathematics Subject Classification: Primary 60G18, 42C40; Secondary 60G20.

Keywords: Elliptic processes, self-similar processes with stationary increments, elliptic pseudo-differential operator, wavelet basis, regularity of sample paths, percolation tree, intermittency. 
In [1] the notion of ellipticity is defined for a Gaussian process indexed by $\mathbb{R}^{d}$. The reproducing kernel Hilbert space of the process is then the energy space of an elliptic operator. An orthonormal basis of this space can be build which is made of wavelets adapted to the elliptic operator. The decomposition of the process on this basis is the first step of a multi-scale analysis which leads to many results, e.g. explicit constants for continuity modulus. In this framework a process is with stationary increments when the symbol of the operator is with constant coefficients. And it is self-similar when the symbol is homogeneous.

In the present paper we plan to study a broad class of stochastic processes indexed by $\mathbb{R}^{d}$ which are self-similar and with stationary increments. No assumption is made about the laws of these processes, but they are required to be elliptic. This is a key point for all the properties to be later studied. So let us first introduce ellipticity with the help of two examples, deterministic and random, and at the same time present a general method to yield elliptic self-similar and with stationary increments (E.S.S.S.I.) processes.

\subsection{Examples and Notations}

As a first example we consider two functions of the real variable $t$

$$
x(t)=\sum_{n \in \mathbb{Z}} b^{-n H} \sin \left(b^{n} t\right), \quad z(t)=\sum_{n \in \mathbb{Z}}\left|t-b^{n}\right|^{-K}-\left|t+b^{n}\right|^{-K},
$$

with parameters $b>1, H, K \in(0,1)$ fixed.

The first function $x$ is known as trigonometric Weierstrass 's function and it is continuous on $\mathbb{R}$. The second one is only almost everywhere defined and locally integrable (w.r.t. the Lebesgue measure on $\mathbb{R}$ ).

We can easily check

$$
b^{-H} x(b t)=x(t), \quad b^{K} z(b t)=z(t), \text { for all } t \in \mathbb{R} .
$$

This means that the functions are invariant by a discrete subgroup of dilations, and such an invariance property is a self-similarity property. Functions $x$ and $z$ belong to a class of self-similar functions which is defined by Jaffard in [7] and which includes the solutions of Iterated System of Functions. It is proved in [7] that all these functions obey the multifractal formalism.

Let us further study the relationship between functions $x$ and $z$. We need here to introduce pseudo-differential operators (p.d.o.) associated to homogeneous symbols $\rho$ on $\mathbb{R}^{d}$, where $d$ is a fixed integer $\geq 1$. The notations $|$.$| ,$ $|\cdot|_{\infty}$ are used respectively for the euclidean and the uniform standard norms. In this paper the symbols that we consider are real valued functions defined 
on $\mathbb{R}^{d}$, symmetric and homogeneous: for some constant $m$

$$
\text { for any } r>0 \text { and any } \xi \in \mathbb{R}^{d}, \rho(r \xi)=r^{m} \rho(\xi) \text {. }
$$

As an alternative definition we can start with a symmetric function $s$ on the unit euclidean sphere $\Sigma_{d-1}$ and set

$$
\rho(\xi)=|\xi|^{m} s\left(\frac{\xi}{|\xi|}\right)
$$

To every symbol $\rho$ is associated a p.d.o. $L[\rho]$ which is given on a suitable domain of regular functions by

$$
L[\rho] \varphi(t)=\int_{\xi \in \mathbb{R}^{d}} \rho(\xi) \widehat{\varphi}(\xi) e^{i t \xi} \frac{d \xi}{(2 \pi)^{d}}
$$

where $t \xi$ denotes the scalar product of $\mathbb{R}^{d}$ and $\widehat{\varphi}=\mathcal{F} \varphi$ the Fourier transform of $\varphi$.

Coming back to functions $x$ and $z$, let us consider the tempered distribution $T$ on $\mathbb{R}$ (here $d=1$ ) given by

$$
T(t)=\sum_{n \in \mathbb{Z}} \sin \left(b^{n} t\right) .
$$

In the distribution meaning its Fourier transform is $\widehat{T}=-i \pi \sum_{n \in \mathbb{Z}}\left(\delta_{b^{n}}-\delta_{-b^{n}}\right)$, if we denote by $\delta_{a}$ the Dirac measure located in $a$. Using a regularisation procedure, we can write functions $x$ and $z$ as

$$
x(t)=\frac{1}{2 \pi}<\widehat{T}, \frac{e^{i t .}-1}{|\cdot|^{H}}>, \quad z(t)=c<\widehat{T},|t-.|^{-K}-|\cdot|^{-K}>
$$

with $c=-2 i \pi^{-1} \sin (K \pi / 2) \Gamma(1-K), \Gamma$ the usual Gamma function. It is now easy to check that $x$ and $z$ are the respective solutions of the following pseudo-differential problems

$$
\left\{\begin{array} { c l } 
{ L [ | \cdot | ^ { H } ] x } & { = T } \\
{ x ( 0 ) } & { = 0 }
\end{array} \quad \left\{\begin{array}{cl}
L\left[|\cdot|^{1+K}\right] z & =-c \widehat{T} \\
z(0) & =0
\end{array}\right.\right.
$$

In the second example we consider stochastic processes. Let us first recall that a process $X=\left\{X(t), \quad t \in \mathbb{R}^{d}\right\}$ is said self-similar with scale parameter $H$ if it satisfies the following identity

$$
\forall \eta>0, \quad\left\{\eta^{-H} X(\eta t), \quad t \in \mathbb{R}^{d}\right\}={ }_{\text {Law }}\left\{X(t), \quad t \in \mathbb{R}^{d}\right\}
$$

where $=_{\text {Law }}$ means equality in law for stochastic processes. 
The standard Fractional Brownian Motion (F.B.M.) with parameter $H$ $(0<H<1)$ is the centered real Gaussian process $B_{H}$ with covariance function

$$
\mathbb{E}\left(B_{H}(s) B_{H}(t)\right)=\frac{1}{2}\left(|s|^{2 H}+|t|^{2 H}-|s-t|^{2 H}\right), \quad s, t \in \mathbb{R}
$$

and the general F.B.M. is $X=c B_{H}, c \in \mathbb{R}$. We say in such a situation that processes $X$ and $B_{H}$ have same laws up to a mulptiplicative constant. From this definition it is easily seen that every F.B.M. is $H$-self-similar and with stationary increments. Let us now show that it is also solution of an elliptic pseudo-differential problem and then an elliptic process. As it is well known (see [11]), the F.B.M. admits various integral representations. A first one, called moving average representation, is

$$
X(t)=c_{1} \int_{y \in \mathbb{R}} \begin{cases}|t-y|^{H-1 / 2}-|-y|^{H-1 / 2} W_{r}(d y) & \text { if } H \neq 1 / 2 \\ 1_{t-y>0}-1_{-y>0} W_{r}(d y) & \text { if } H=1 / 2\end{cases}
$$

where $c_{1} \in \mathbb{R}$ and $W_{r}$ is the real Gaussian white noise, i.e. the real centered Gaussian random measure which satisfies for every $A, B$ bounded Borel subsets of $\mathbb{R}$,

$$
\mathbb{E}\left(W_{r}(A) W_{r}(B)\right)=\int_{y \in \mathbb{R}} 1_{A}(y) 1_{B}(y) d y .
$$

A second one is the so called harmonic representation

$$
X(t)=c_{2} \int_{\xi \in \mathbb{R}} \frac{\exp (i t \xi)-1}{|\xi|^{H+1 / 2}} \widehat{W}_{r}(d \xi)
$$

where $c_{2} \in \mathbb{R}$. Using the last representation it can be seen that the Fractional Brownian Motion is the solution of the following elliptic problem

$$
L\left[|.|^{H+1 / 2}\right] X=2 \pi c_{2} W_{r}, \quad X(0)=0 .
$$

N.B.: It must be noticed that the Fourier transform of $W_{r}$ is the standard centered complex Gaussian white noise.

The link between the two representations is a Plancherel identity, the kernel in the second integral being the Fourier transform of the kernel in the first one.

In view of the relation between functions $x, z$ which are the solutions of equations (1.5) we can look for the solution of the following elliptic problem

$$
L\left[|\cdot|^{K+1 / 2}\right] Z=\widehat{W}_{r}, \quad Z(0)=0 .
$$


In the case where $0<K<1$ the following process

$$
Z(t)=\mathcal{R} e \int_{\xi \in \mathbb{R}} \frac{\exp (i t \xi)-1}{|\xi|^{K+1 / 2}} W_{r}(d \xi)
$$

is well defined and is solution of (1.8).

It is easily checked that $Z$ is $K$ - self-similar and with stationary increments. As the Gaussian white noise is invariant with respect to the Fourier transform we find that $Z$ is a version of the F.B.M. with parameter $K$. So that the laws of $X$ and $Z$ are the same and there occurs no new process.

However, it is important to notice that scaling one process amounts to inverse scaling for the other. The same was already true for functions $x$ and $z$, see (1.2). As a consequence, even when $H=K$, a non trivial linear combination

$$
Y=\alpha X+\beta Z
$$

( $\alpha \neq 0$ and $\beta \neq 0$ ) is no longer self-similar. This deserves a special definition that we now give in the general case of processes indexed by $\mathbb{R}^{d}$.

Definition 1.1 We say a pair $X, Z$ of stochastic processes enjoys Inverse Scaling Invariance property with parameter $(H, K)$-or I.S.I. $(H, K)$ - when

$$
\left\{\left(\eta^{-H} X(\eta t), \eta^{K} Z(y / \eta)\right) ; t, y \in \mathbb{R}^{d}\right\}={ }_{L a w}\left\{(X(t), Z(y)) ; t, y \in \mathbb{R}^{d}\right\}
$$

$\forall \eta>0$. The notation will be shortened in I.S.I.(H) in the case $H=K$.

Remark 1.1 If the pair $(X, Z)$ satisfies the I.S.I. $(H, K)$ property then each process is separately self-similar with respective parameter $H, K$ and any linear combination $\alpha X+\beta Z$ satisfies

$$
\forall \eta>0, \quad \alpha \frac{1}{\eta^{H}} X(\eta .)+\beta \eta^{K} Z\left(\frac{\dot{\eta}}{\eta}\right)={ }_{L a w} \alpha X(.)+\beta Z(.) .
$$

\subsection{Purposes}

Motivated by the previous examples we shall consider the following situation: let $\rho, \rho_{*}$ some homogeneous symbols as given in (1.3) with respective degrees

$$
m=\frac{d}{\alpha}+H, m_{*}=\frac{d}{\alpha^{*}}+K
$$

where $H, K$ are in $(0,1)$ and $\alpha, \alpha^{*}$ are conjugate exponents,

$$
\frac{1}{\alpha}+\frac{1}{\alpha^{*}}=1
$$


Let $L[\rho], L\left[\rho_{*}\right]$ the associated p.d.o., as in (1.4) and let $M$ be a stochastic measure on $\mathbb{R}^{d}$ in a sense which we will precise in section (3.1).

We plan to show that there exists a unique pair $X, Z$ of processes indexed by $\mathbb{R}^{d}$ which solves

$$
\left\{\begin{array} { c } 
{ L [ \rho ] X = M } \\
{ X ( 0 ) = 0 }
\end{array} \quad \left\{\begin{array}{c}
L\left[\rho_{*}\right] Z=\widehat{M} \\
Z(0)=0
\end{array}\right.\right.
$$

in the distribution meaning. Then we study conditions on measure $M$ which involve for processes $X$ and $Z$ the two following properties : I.S.I. $(H, K)$ and stationarity of increments.

As an important class of $M$ 's we find the family of independently scattered stochastic measures,

$$
\begin{aligned}
& M\left(B_{i}\right), i=1, \ldots, n \text { mutually independent if } \\
& \qquad B_{i}, i=1, \ldots, n \text { are pairwise disjoints, }
\end{aligned}
$$

with $S \alpha S$ (=symmetric $\alpha$-stable) laws. The two properties are linked, see Remark 4.2. In this case processes $X$ and $Z$ belong to the class of $S \alpha S$ processes, which is extensively studied: see [10], [15] and the book [13] with all its references. In has been shown that the the Gaussian case $(\alpha=2)$ radically differs from the non-Gaussian one: only in the former case are the laws of processes $X, Z$ equal up to a multiplicative constant. This can be proved after studying the behaviour of the codifference functions toward infinity. The method we use in the present paper shed another light on this result, by using the elliptic operators which are associated to these processes.

In the next section we study the regularity of E.S.S.S.I. processes. It is shown that under some condition on the parameters almost all their paths are continuous. The proof rests here on an appropriate decomposition of the process, using a specially build wavelet basis in the spirit of [1].

In the last section we want to show how easy such a decomposition can lead to new E.S.S.S.I. processes. The main idea there is to consider a percolation forest in connection with the wavelet decomposition. These processes can be useful models for physical systems which exhibit both properties: 1) intermittency (which corresponds to the forest), 2) self-similarity and stationary increments (this corresponds to the wavelet basis). It will be clear that the processes we obtain in this case are with non stable laws. 


\section{Elliptic equations, notations and first results}

\subsection{Fourier transform of homogeneous functions}

Let us recall some properties of the Fourier transform $\mathcal{F}$ we shall need. We denote by $\mathcal{S}\left(\mathbb{R}^{d}\right)$ the space of functions which are smooth on $\mathbb{R}^{d}$ and rapidly decaying at infinity. This space is equipped with its usual locally convex topology. We denote by $\mathcal{S}^{\prime}\left(\mathbb{R}^{d}\right)$ the space of tempered distributions, its dual. The Banach space of functions which are of $p$-power integrable (resp. essentially bounded) w.r.t. the Lebesgue measure is denoted by $L^{p}\left(\mathbb{R}^{d}\right)$ when $1 \leq p<\infty$ (resp. $p=\infty)$.

If $T$ is a integrable function or a tempered distribution on $\mathbb{R}^{d}$ we know

$$
\mathcal{F}(T(\eta .))(\xi)=|\eta|^{-d} \widehat{T}\left(\frac{\xi}{\eta}\right), \quad \mathcal{F}(T(y+.))(\xi)=\exp (-i y \xi) \widehat{T}(\xi),
$$

are valid $\forall \eta \neq 0, \forall y \in \mathbb{R}^{d}$.

If $\gamma \in(-d, 0)$ and $\xi \neq 0$ we have

$$
\mathcal{F}\left(|.|^{\gamma}\right)(\xi)=c(\gamma, d) \frac{1}{|\xi|^{d+\gamma}} \quad \text { with } c(\gamma, d)=2^{d+\gamma} \pi^{d / 2} \Gamma\left(\frac{d+\gamma}{2}\right) / \Gamma\left(\frac{-\gamma}{2}\right) .
$$

This result can be extended to every $\gamma \notin-d-2 \mathbb{C}$ if we replace ordinary power functions by pseudo-functions $P f|.|^{\gamma}$, see Schwartz [14].

As a more general result, we know that the Fourier transform of a $\gamma$ homogeneous distribution is a $-(d+\gamma)$-homogeneous distribution. Let $r$ a measurable function defined on the unit sphere $\Sigma_{d-1}$ which is (1) real-valued (2) bounded and (3) symmetric and $\gamma \in(-d, 0)$; there exists a smooth function $q$ on $\Sigma_{d-1}$ with the same properties (1), (2), (3) such that, when $\xi \neq 0$,

$$
\mathcal{F}\left(|\cdot|^{\gamma} r\left(\frac{\cdot}{|.|}\right)\right)(\xi)=c(\gamma, d) \frac{1}{|\xi|^{d+\gamma}} q\left(\frac{\xi}{|\xi|}\right) .
$$

The same is valid for a general $\gamma$ by using pseudo-functions, see [14].

We set $\tau_{\gamma}(r)=c(\gamma, d) q$, which defines a positive linear transformation for real functions on the unit sphere $\Sigma_{d-1}$.

\subsection{Ellipticity hypothesis. Kernels}

We consider parameters $\beta$ and $H$ such that

$$
\beta \geq 1, \quad 0<H<1
$$

and a real-valued symmetric measurable function $s$ on $\Sigma_{d-1}$ which satisfies an "ellipticity" condition

$$
\exists c>0, \quad c \leq s(t) \leq \frac{1}{c} \quad \forall t \in \Sigma_{d-1} .
$$


Then we set $q=c(-H-d / \beta, d) \tau_{-H-d / \beta}(1 / s)$ and define two kernels $R_{\beta, s}$ and $K_{\beta, s}$ by

$$
\begin{gathered}
R_{\beta, s}(t, \xi):=\frac{\exp (i t \xi)-1}{|\xi|^{\frac{d}{\beta}+H} s(\xi /|\xi|)(2 \pi)^{d}} \\
K_{\beta, s}(t, y):=|t-y|^{H-\frac{d}{\beta}} q((t-y) /|t-y|)-|-y|^{H-\frac{d}{\beta}} q(-y /|-y|) .
\end{gathered}
$$

Lemma 2.1 We suppose (2.4), (2.5) hold, $1 \leq \beta<\infty$ and $t \in \mathbb{R}^{d}$; then both functions $K_{\beta, s}(t,$.$) and R_{\beta, s}(t,$.$) are with \beta$-power integrable w.r.t. the Lebesgue measure. As functions of they are continuous with respect to the $L^{\beta}\left(\mathbb{R}^{d}\right)$-norm. Furthermore

$$
\forall(t, \xi) \in \mathbb{R}^{d} \times \mathbb{R}^{d} \backslash\{0\}, \quad \mathcal{F}\left(K_{\beta, s}(t, .)\right)(\xi)=R_{\beta^{*}, s}(t, \xi) .
$$

Proof. Let us begin with $K_{\beta, s}(t, y)$.

We set $c_{0}=\max _{|z|=1}|q(z)|$ and $c_{1}=\max _{|v|=|w|=1, v \neq w}|q(w)-q(v)| /|w-v|$ which are finite constants as we know $q$ is smooth on $\Sigma_{d-1}$ and we set also $\rho=|y|, \quad y=\rho u, \mu=H-d / \beta$. If $|t| / \rho \leq 1 / 2$ we get

$$
\begin{aligned}
\left|K_{\beta, s}(t, y)\right| & =\rho^{\mu}\left(\left|\frac{t}{\rho}-u\right|^{\mu} q\left(\frac{t / \rho-u}{|t / \rho-u|}\right)-q\left(\frac{-u}{|-u|}\right)\right) \\
& \leq \rho^{\mu}\left(c_{0}|| \frac{t}{\rho}-\left.u\right|^{\mu}-1\left|+2 c_{1}\right| \frac{t}{\rho} \mid\right) \leq c_{2} \rho^{\mu} \frac{|t|}{\rho}=c_{2} \rho^{H-d / \beta-1}|t|
\end{aligned}
$$

and then

$$
\int_{|y| \geq 2|t|}\left|K_{\beta, s}(t, y)\right|^{\beta} d y \leq c_{3}|t|^{\beta} \int_{\rho=2|t|}^{+\infty} \rho^{\beta H-d-\beta+d-1} d \rho
$$

which is finite because $H<1$.

The $\beta$-integrability of $K_{\beta, s}(t,$.$) around 0$ and around $t$ is easily reduced to the $\beta$-integrability of $y \mapsto G(y):=1_{|y| \leq 1}|y|^{H-d / \beta} q(y /|y|)$. By using polar decomposition this last property is equivalent to condition

$$
\int_{0}^{1} r^{H \beta-d} r^{d-1} d r<+\infty
$$

This is satisfied if and only if $\beta H>0$, which is true since $H>0$.

Let us now prove that $t \mapsto K_{\beta, s}(t,$.$) is continuous at 0$ w.r.t. the $L^{\beta}\left(\mathbb{R}^{d}\right)$-norm. Using the translation invariance of Lebesgue measure we need only to work at $t=0$. We can split the integral in two parts, according to $|y| \leq 1$ or $|y|>1$. As $G$ belongs to $L^{\beta}\left(\mathbb{R}^{d}\right)$ and $\beta<\infty$, we know the 
function $t \in \mathbb{R}^{d} \mapsto G\left(t-\right.$.) is continuous w.r.t. the $L^{\beta}\left(\mathbb{R}^{d}\right)$-norm and this gives the continuity property for the first part. The second part is controlled with the help of inequality (2.9) so that we get the desired result.

The same methods can be applied to $R_{\beta, s}(t,$.$) . It belongs to the space$ $L^{\beta}\left(\mathbb{R}^{d}, d t\right)$ when conditions

$$
\int_{0}^{1} r^{\beta-H \beta-d} r^{d-1} d r<\infty \text { and } \int_{1}^{+\infty} r^{-H \beta-d} r^{d-1} d r<\infty
$$

are satisfied, and we get the same conclusions as above.

To show that (2.8) holds we assume first that $H \beta<d$. We are then in the case where $\gamma=H-d / \beta$ (the parameter used for $K_{\beta, s}(t,$.$) ) and$ $\gamma^{\prime}=-H-d / \beta^{*}=d-\gamma$ (the parameter used for $\left.R_{\beta^{*}, s}(t,).\right)$ both belong to $(-d, 0)$, that is the case where functions are locally integrable. Then the formula (2.8) is a direct application of (2.3) with equality between functions on $\mathbb{R}^{d} \backslash\{0\}$. The result extends as usual to the general case in the framework of pseudo-functions. But using the $\beta$-integrability property just proved above it is also an ordinary functional equality.

We denote by $\mathcal{R}_{\beta, s}$ and $\mathcal{K}_{\beta, s}$ the operators with kernels $R_{\beta, s}(t, \xi)$ and $K_{\beta, s}(t, y)$ respectively,

$$
\mathcal{R}_{\beta, s} f(t)=\int_{\mathbb{R}^{d}} R_{\beta, s}(t, \xi) f(\xi) d \xi, \quad \mathcal{K}_{\beta, s} f(t)=\int_{\mathbb{R}^{d}} K_{\beta, s}(t, y) f(y) d y
$$

In the general case the kernels $R_{\beta, s}$ and $K_{\beta, s}$ are distributions on $\mathbb{R}^{d}$ and we consider integrals of (2.10) in the distribution meaning.

\subsection{Elliptic pseudo-differential problems}

In the sequel $\alpha$ is a positive number such that $1<\alpha \leq 2$, $\alpha^{*}$ its conjugate exponent and $H, K$ are in $(0,1)$. We consider a function $s$ which satisfies ellipticity condition (2.5) and symbols $\rho, \rho_{*}$ associated with $s$ as in (1.3) with respective degree $m=d / \alpha+H, m_{*}=d / \alpha^{*}+K$. For the p.d.o. associated with the symbols $\rho, \rho_{*}$ we use the simplified notations $L_{\alpha}$ and $L_{\alpha^{*}}$. We shall also shorten notations of previously defined kernels and operators and simply write $R_{\beta}, \mathcal{R}_{\beta}, K_{\beta}, \mathcal{K}_{\beta}$, forgetting to write the dependance on $s$.

We consider the following two problems:

- to find functions $u$ such that

$$
\left(P_{*}\right) \quad L_{\alpha^{*}} u(t)=(2 \pi)^{-d} \widehat{f}(-t), \quad u(0)=0
$$

- to find functions $v$ such that

$$
\text { (P) } L_{\alpha} v(t)=g(t), \quad v(0)=0 .
$$


We look for solutions in some Banach spaces which we now define. Let us denote by $\Delta$ be the $d$-dimensional laplacian and by $W_{0}^{r, \beta}$ the Sobolev homogeneous space

$$
W_{0}^{r, \beta}=\left\{u,(-\Delta)^{r / 2} u \in L^{\beta}\left(\mathbb{R}^{d}\right), D^{\mu} u(0)=0 \text { for } \mu \in \mathbb{C}^{d},|\mu| \leq r-\frac{d}{\beta}\right\}
$$

equipped with the seminorm $\|u\|_{r, \beta}=\left(\int_{\mathbb{R}^{d}}\left|(-\Delta)^{r / 2} u(t)\right|^{\beta} d t\right)^{1 / \beta}$.

Lemma 2.2 Let $\alpha \in(1,2], H \in(0,1)$ and $m=d / \alpha+H, m_{*}=d / \alpha^{*}+H$. Then $W_{0}^{m, \alpha}$ and $W_{0}^{m_{*}, \alpha^{*}}$ are Banach spaces and the condition in 0 reduces to $u(0)=0$.

This Banach space property is valid for $W_{0}^{r, \beta}$ under general conditions, as explained in the book of Meyer $([12])$, chapter VI. The case which is considered here satisfies $0<r-d / \beta<1$ so that no condition for derivatives of order $\geq 1$ in 0 is needed.

Once the spaces are well defined we get existence and unicity results as given in the following theorem.

Theorem 2.1 We suppose that $1<\alpha \leq 2, f, g \in L^{\alpha}\left(\mathbb{R}^{d}\right)$, and $s$ satisfies (2.5). Then

(i) $\mathcal{R}_{\alpha^{*}}(f)$ is well defined and is the unique element in $W_{0}^{m_{*}, \alpha^{*}}$ solution of problem $\left(P_{*}\right)$.

(ii) $\mathcal{R}_{\alpha}(\widehat{g})$ is well defined and is the unique element in $W_{0}^{m, \alpha}$ solution of problem $(P)$.

(iii) Furthermore we have Plancherel like formulas

$$
\mathcal{R}_{\alpha^{*}}(f)=\mathcal{K}_{\alpha}(\widehat{f}), \quad \mathcal{R}_{\alpha}(\widehat{g})=\mathcal{K}_{\alpha^{*}}(g) .
$$

Proof. Using the integrability result of Lemma 2.1 we can assert that for every $f \in L^{\alpha}\left(\mathbb{R}^{d}\right)$ the following integral makes sense and defines a continuous function $u_{f}$ of the $t$ variable

$$
\int_{\xi \in \mathbb{R}^{d}} R_{\alpha^{*}}(t, \xi) f(\xi) d \xi=: u_{f}(t)
$$

This function $u_{f}$ clearly vanishes at 0 . To see that $u_{f} \in W_{0}^{m_{*}, \alpha^{*}}$ it remains to prove

$$
(-\Delta)^{m_{*} / 2} u_{f} \in L^{\alpha^{*}}\left(\mathbb{R}^{d}\right) .
$$


Let us first suppose that $f \in L_{0}^{\alpha}\left(\mathbb{R}^{d}\right)$, the subspace of functions $\alpha$-integrable which vanish on some neighbourhood of 0 . In this case $u_{f}(t)=U(t)-U(0)$, with $U:=\mathcal{F}^{-1}\left(\frac{f(.)}{|\cdot|^{m_{*}} s(. /|\cdot|)}\right)$, therefore

$$
\mathcal{F}\left(u_{f}\right)=\frac{f(.)}{|.|^{m_{*}} s(. /|.|)}-(2 \pi)^{d} U(0) \delta_{0}
$$

and then

$$
<|.|^{m_{*}} \mathcal{F}\left(u_{f}\right), \varphi>=<\frac{f(.)}{s(. /|.|)}, \varphi>
$$

for every $\varphi \in \mathcal{S}\left(\mathbb{R}^{d}\right)$. This means that in the distribution meaning

$$
(-\Delta)^{m_{*} / 2} u_{f}=(2 \pi)^{-d} \mathcal{F}\left(\frac{f}{s(. /|.|)}\right)
$$

As $\alpha \leq 2$ we know that $\mathcal{F} L^{\alpha}\left(\mathbb{R}^{d}\right) \subset L^{\alpha^{*}}\left(\mathbb{R}^{d}\right)$, see [16], so that we get (2.13).

Recalling the definition of the operator $L_{\alpha^{*}}$ and using previous equalities we get also

$$
\begin{aligned}
\mathcal{F}\left(L_{\alpha^{*}} u_{f}\right)(\xi) & =s(\xi /|\xi|)|\xi|^{m_{*}} \mathcal{F}\left(u_{f}\right)(\xi) \\
& =f(\xi)
\end{aligned}
$$

which means that $u_{f}$ solves the problem $\left(P_{*}\right)$.

As $L_{0}^{\alpha}\left(\mathbb{R}^{d}\right)$ is a dense subspace of the Banach space $L^{\alpha}\left(\mathbb{R}^{d}\right)$ the result holds for every $f \in L^{\alpha}\left(\mathbb{R}^{d}\right)$.

The second part of Theorem 2.1 deals with problem $(P)$. Here we use $\widehat{g} \in L^{\alpha^{*}}\left(\mathbb{R}^{d}\right)$ and $R_{\alpha}(t,.) \in L^{\alpha}\left(\mathbb{R}^{d}\right)$ (see Lemma 2.1). Then

$$
v_{g}(t):=\int_{\xi \in \mathbb{R}^{d}} R_{\alpha}(t, \xi) \widehat{g}(\xi) d \xi
$$

is a well defined continuous function on $\mathbb{R}^{d}$, vanishing at 0 . That the function $v_{g}$ belongs to $W_{0}^{m, \alpha}$ and gives a solution to problem $(P)$ can be proved exactly as above for $\left(P_{*}\right)$, working first in the case where $g$ vanishes in 0 , then using a density argument.

The identities (2.12) are now deduced from the result (2.8) of Lemma 2.1 by using the well known Plancherel formulas.

To complete the proof we must show that the solution of problem $\left(P_{*}\right)$ (resp. $(P)$ ) is unique. Using equality $(2.14)$ and the definition of space $W_{0}^{m_{*}, \alpha^{*}}$ we see that the kernel of operator $L_{\alpha^{*}}$ reduces to the nullspace. This implies the required unicity for problem $\left(P_{*}\right)$. The same method applies to problem $(P)$. 


\section{Elliptic stochastic processes}

\subsection{Generalized processes}

The notion of linear process has been studied by Gelfand [6]. When the parameter set is $\mathcal{S}\left(\mathbb{R}^{d}\right)$ a linear process is called a generalized process or a random distribution. We use two notations

$$
\varphi \mapsto Y(\varphi, \omega) \quad \text { or } \quad \varphi \mapsto<Y(\omega), \varphi>
$$

for a generalized process $Y: \mathcal{S}\left(\mathbb{R}^{d}\right) \mapsto L^{0}(\Omega, \mathbb{P})$. The Fourier transform $\hat{Y}$ of a generalised process $Y$ is defined as usual by duality. It is also denoted by $\mathcal{F} Y$.

In order to define invariance properties we introduce several operators $S_{\mu}, T_{t}, U_{\xi}$ by setting

$$
\forall y \in \mathbb{R}^{d}, S_{\mu}(f)(y)=f(\mu y), T_{t}(f)(y)=f(y-t), \quad U_{\xi}(f)(y)=e^{i \xi \cdot y} f(y)
$$

given $t, \xi \in \mathbb{R}^{d}$ and $\mu \in \mathbb{R}$.

A linear process $Y$ is said to enjoy

1. (SM1), stationarity, if $\forall t \in \mathbb{R}^{d}$

$$
\operatorname{Law}\left(\left\{Y\left(T_{t} \varphi\right), \varphi \in \mathcal{S}\left(\mathbb{R}^{d}\right)\right\}\right)=\operatorname{Law}\left(\left\{Y(\varphi), \varphi \in \mathcal{S}\left(\mathbb{R}^{d}\right)\right\}\right),
$$

2. (SM2), unitarity, if $\forall \xi \in \mathbb{R}^{d}$

$$
\operatorname{Law}\left(\left\{Y\left(U_{\xi} \varphi\right), \varphi \in \mathcal{S}\left(\mathbb{R}^{d}\right)\right\}\right)=\operatorname{Law}\left(\left\{Y(\varphi), \varphi \in \mathcal{S}\left(\mathbb{R}^{d}\right)\right\}\right),
$$

3. (SM3), scaling property of order $k$, if $\forall \eta \neq 0$

$$
\operatorname{Law}\left(\left\{Y\left(S_{\eta} \varphi\right), \varphi \in \mathcal{S}\left(\mathbb{R}^{d}\right)\right\}\right)=\operatorname{Law}\left(\left\{|\eta|^{-k-d} Y(\varphi), \varphi \in \mathcal{S}\left(\mathbb{R}^{d}\right)\right\}\right)
$$

It should be noticed that if $Y$ satisfies scaling property of order $K$ then $\widehat{Y}$ satisfies scaling property of order $d-K$.

Definition 3.1 Let $\nu$ be some deterministic measure on $\left(\mathbb{R}^{d}, \mathcal{R}^{d}\right)$ and $\beta \geq 1$, $p \geq 1$. A generalized process $M$ is said of type $(\beta, p)$ with control measure $\nu$ if

$$
\exists c>0, \forall \varphi \in \mathcal{S}\left(\mathbb{R}^{d}\right), \quad\|M(\varphi)\|_{L^{p}(\Omega, \mathbb{P})} \leq c\|\varphi\|_{L^{\beta}\left(\mathbb{R}^{d}, d \nu\right)} .
$$


Such a $M$ can be extended as a linear process on the space $L^{\beta}\left(\mathbb{R}^{d}, d \nu\right)$ and in particular $M\left(1_{A}\right)$ makes sense when $\nu(A)<\infty$ : the notation $M(A)=$ $M\left(1_{A}\right)$ is used and $M$ is called a random measure. The following countably additivity property is satisfied: for every sequence $\left(A_{i}\right)_{i \in \mathbb{N}}$ of Borel sets which are pairwise disjoints and such that $\nu\left(\cup_{i \in \mathbb{N}} A_{i}\right)<\infty$ we have

$$
M\left(\cup_{i \in \mathbb{N}} A_{i}\right)=\sum_{i \in \mathbb{N}} M\left(A_{i}\right),
$$

with convergence in $L^{p}(\Omega, d \mathbb{P})$ sense.

A random measure is said independently scattered when for every sequence of pairwise disjoint sets $\left(A_{i}\right)_{i \in \mathbb{N}}$ the corresponding random sequence $\left(M\left(A_{i}\right), \quad i \in \mathbb{C}\right)$ is an independent sequence. In the present paper this independence property is not necessarily required.

We shall denote by $S L^{\beta, p}\left(\mathbb{R}^{d}, \nu\right)$ the space of generalized processes $M$ for which (3.1) is true. We define the norm of $M \in S L^{\beta, p}(\nu)$ as the best constant $c$ in (3.1). If $\nu$ is the Lebesgue measure on $\mathbb{R}^{d}$ we simply write $S L^{\beta, p}$.

\subsection{The stochastic problems}

Let us now introduce the spaces of stochastic processes which are useful for the setting of our elliptic stochastic problems. We recall $m=d / \alpha+H$, $m_{*}=d / \alpha^{*}+K$ where $H, K$ are in $(0,1), \alpha, \alpha^{*}$ are conjugate exponents, $\alpha \in(1,2]$, see (1.11). As $H, K$ are in $(0,1)$ the conditions in 0 are order zero conditions.

Definition 3.2 We shall say that a generalized stochastic process $X$ belongs to $S W_{0}^{m, \alpha, p}\left(\mathbb{R}^{d}, \nu\right)$ if

$$
(-\Delta)^{m / 2} X \in S L^{\alpha^{*}, p}\left(\mathbb{R}^{d}, \nu\right) \quad \text { and } \quad X(0)=0
$$

where we define the vanishing in 0 condition by using Gaussian densities $g_{\sigma}(x)=(\sigma \sqrt{2 \pi})^{-d} \exp \left(-\frac{x^{2}}{2 \sigma^{2}}\right)$,

$$
X(0)=0 \Longleftrightarrow \lim _{\sigma \rightarrow 0}<X, g_{\sigma}>=0 \quad \text { in } L^{0}(\Omega, \mathbb{P}) .
$$

We consider the two equations

$$
\begin{gathered}
\left(S P_{*}\right) \quad L_{\alpha^{*}} X=M, \quad X(0)=0, \\
(S P) \quad L_{\alpha} Z=\widehat{M}, \quad Z(0)=0,
\end{gathered}
$$

where $M$ is a random measure and $L_{\alpha}, L_{\alpha^{*}}$ are elliptic operators. The symbols are homogeneous with respective degrees $m, m_{*}$ which satisfy (1.11) and they depend on a function $s$ which satisfy (2.5). 
Theorem 3.1 We suppose $M$ is a stochastic measure of type $(\alpha, p)$ with control measure $\nu=$ Lebesgue measure on $\mathbb{R}^{d}$.

There exists a unique pair $(X, Z)$ in $S W_{0}^{m_{*}, \alpha_{*}, p} \times S W_{0}^{m, \alpha, p}$ such that $X$ (resp. Z) solves $\left(S P_{*}\right)$ (resp. $\left.(S P)\right)$ which is given by

$$
X=\mathcal{R}_{\alpha_{*}}(\widehat{M}), \quad Z=\mathcal{R}_{\alpha}(M)
$$

Furthermore we have Plancherel like formulas

$$
\mathcal{R}_{\alpha^{*}}(\widehat{M})=\mathcal{K}_{\alpha}(M), \quad \mathcal{R}_{\alpha}(M)=\mathcal{K}_{\alpha^{*}}(\widehat{M})
$$

in the distribution meaning.

Proof. From Lemma $(2.1)$ we know that $\widehat{R_{\alpha_{*}}}(x,)=.K_{\alpha}(x,.) \in L^{\alpha}\left(\mathbb{R}^{d}\right)$ and since the generalised process $M$ is of type $(p, \alpha)$ we get a well defined ordinary process $X$ by setting

$$
X_{x}=<\widehat{M}, R_{\alpha^{*}}(x, .)>=<M, \widehat{R_{\alpha^{*}}}(x, .)>.
$$

Let us now prove a Fubini like result: we have equality

$$
<\mathcal{R}_{\alpha_{*}}(\widehat{M}), \varphi>=<M, \mathcal{R}_{\alpha_{*}} \widehat{\varphi}>,
$$

as processes indexed by $\varphi \in L^{\alpha}\left(\mathbb{R}^{d}\right)$. This is a consequency of the symmetry of the operator $\mathcal{R}_{\alpha^{*}} \circ \mathcal{F}$, which is proved as soon as we get

$$
<\mathcal{R}_{\alpha^{*}} \widehat{\varphi}, \psi>=<\varphi, \mathcal{R}_{\alpha^{*}} \widehat{\psi}>
$$

for $\varphi, \psi$ running through a dense subspace of $L^{\alpha}\left(\mathbb{R}^{d}\right)$. Let us consider $\mathcal{S}_{0}$ the set of functions $\psi \in \mathcal{S}\left(\mathbb{R}^{d}\right)$ with vanishing mean, $\int_{\mathbb{R}^{d}} \psi(t) d t=0$. As $\alpha>1$, $\mathcal{S}_{0}$ is a dense subspace of $L^{\alpha}\left(\mathbb{R}^{d}\right)$.

For every $\varphi \in \mathcal{S}_{0}$ we have

$$
\mathcal{R}_{\alpha^{*}} \widehat{\varphi}(x)=(2 \pi)^{-d} \int_{\mathbb{R}^{d}} \frac{e^{i x \xi}-1}{|\xi|^{m_{*}} s(\xi /|\xi|)} \widehat{\varphi}(\xi) d \xi=(2 \pi)^{-d} \mathcal{F}\left(\frac{\widehat{\varphi}}{|\cdot|^{m_{*} S}}\right)(-x)-c
$$

with $c$ a constant. And thus for every $\varphi, \psi \in \mathcal{S}_{0}$ we get

$$
\begin{aligned}
<\mathcal{R}_{\alpha^{*}} \mathcal{F} \varphi, \psi>=<\frac{\widehat{\varphi}}{|.|_{*} S}-c \delta_{0}, \widehat{\psi}> \\
\quad=\int_{\mathbb{R}^{d}} \frac{\widehat{\varphi}(\xi) \widehat{\psi}(\xi)}{|\xi|^{m_{*}} s(\xi /|\xi|)} d \xi-c \int_{\mathbb{R}^{d}} \psi(t) d t,=\int_{\mathbb{R}^{d}} \frac{\widehat{\varphi}(\xi) \widehat{\psi}(\xi)}{|\xi|^{m_{*}} s(\xi /|\xi|)} d \xi
\end{aligned}
$$

an expression which is symmetric in $\varphi, \psi$. The equality (3.3) is proved. 
Let us see that $X \in S W_{0}^{m_{*}, \alpha_{*}, p}$. The vanishing in 0 condition is obvious $(K(0, y) \equiv 0)$ so it remains to prove

$$
\mathbb{E}\left|<X,(-\Delta)^{m_{*} / 2} \psi>\right|^{p} \leq c|\psi|_{L^{\alpha}\left(\mathbb{R}^{d}\right)}^{p} .
$$

It is sufficient to prove (3.5) for functions $\psi$ in $\mathcal{S}\left(\mathbb{R}^{d}\right)$ such that

$$
\int_{\mathbb{R}^{d}} \frac{\mathcal{F} \psi(\xi)}{s(\xi /|\xi|)} d \xi=0
$$

the general result will be true by a density argument (when $\alpha>1$ ). For such a $\varphi$ we have

$$
\begin{aligned}
& <X,(-\Delta)^{m_{*} / 2} \psi>=\int_{\mathbb{R}^{d}} \mathcal{R}_{\alpha_{*}}\left(|\cdot|^{m_{*}} \mathcal{F} \psi\right) d M \\
& \quad=\int_{\mathbb{R}^{d}}\left(\int_{\mathbb{R}^{d}} \frac{e^{i t \xi}-1}{(2 \pi)^{d} s(\xi /|\xi|)} \mathcal{F} \psi(\xi) d \xi\right) M(d t)=\int_{\mathbb{R}^{d}} H \psi(t) M(d t)
\end{aligned}
$$

where $H=o p(1 / S)$ is the pseudo-differential operator with symbol $1 / s(\xi /|\xi|)$. Since $S$ is bounded by positive constants this operator is bounded in the $L^{\alpha}\left(\mathbb{R}^{d}\right)$ norm. As $M$ is of type $(p, \alpha)$ we get for some constant $c$

$$
\left|\int_{\mathbb{R}^{d}} H \psi d M\right|_{L^{p}(\Omega)} \leq c|\psi|_{L^{\alpha}\left(\mathbb{R}^{d}\right)}
$$

which gives the result (2.5).

In order to show that $X=\mathcal{R}_{\alpha^{*}} \mathcal{F} M$ solves problem $\left(S P_{*}\right)$ it is enough to prove

$$
\forall \varphi \in \mathcal{S}\left(\mathbb{R}^{d}\right),<L_{\alpha^{*}} \mathcal{R}_{\alpha_{*}} \mathcal{F} M, \varphi>=<M, \varphi>.
$$

But this is an easy consequency of Theorem 2.1 where we have seen that

$$
\forall \varphi \in \mathcal{S}\left(\mathbb{R}^{d}\right), L_{\alpha^{*}} \mathcal{R}_{\alpha_{*}} \mathcal{F} \varphi=\varphi
$$

The solution of problem $\left(S P_{*}\right)$ is unique: in fact if $X \in S W_{0}^{m_{*}, \alpha_{*}, p}(\mu)$ and satisfies

$$
\forall \varphi \in \mathcal{S}\left(\mathbb{R}^{d}\right),<L_{\alpha^{*}} X, \mathcal{F} \varphi>=0
$$

we obtain

$$
\forall \varphi \in \mathcal{S}\left(\mathbb{R}^{d}\right), \int_{\mathbb{R}^{d}} \varphi(\xi)|\xi|^{m_{*}} s(\xi /|\xi|) \mathcal{F} X(\xi) d \xi=0
$$

and as $X(0)=0$ we can conclude $X=0$ in $S W_{0}^{m_{*}, \alpha_{*}, p}$.

The previous arguments can be adapted to solve problem $(S P)$ and therefore the corresponding proof is omitted. The first Plancherel formula is a direct consequence of equation (3.3). The second one is similar.

Remark 3.1 The previous results can be extended to measures $\nu$ which are equivalent to Lebesgue measure. 


\section{Self-similar Processes}

\subsection{Condition for self-similarity}

We are looking for scaling properties of processes $X, Z$ which are respective solutions of $\left(S P_{*}\right),(S P)$. As these processes are linked by the stochastic measure $M$ it seems more relevant to study the Inverse Scaling Invariance $(H, K)$ property.

Theorem 4.1 Let us suppose that hypotheses of Theorem 3.1 hold. The solution $X, Z$ of $\left(S P_{*}\right),(S P)$ is Inverse Scale Invariant with parameter $H, K$ and also with stationary increments if the linear process $M$ satisfies conditions SM1 to SM3 with $k=-d / \alpha^{*}$.

Proof. By hypothesis the random measure $M$ satisfies (SM3) with $k=$ $-d / \alpha^{*}$

$\forall \mu>0,\left(\int_{\mathbb{R}^{d}} \varphi\left(\frac{\xi}{\mu}\right) M(d \xi), \varphi \in \mathcal{S}\left(\mathbb{R}^{d}\right)\right)={ }_{L a w}\left(\mu^{d / \alpha} \int_{\mathbb{R}^{d}} \varphi(\xi) M(d \xi), \varphi \in \mathcal{S}\left(\mathbb{R}^{d}\right)\right)$.

Using a regularisation argument we get the following equality in law of ordinary processes

$$
\forall \mu>0,\left(\int_{\mathbb{R}^{d}} f_{t}(\xi / \mu) M(d \xi), t \in \mathbb{R}^{d}\right)={ }_{L a w}\left(\mu^{d / \alpha} \int_{\mathbb{R}^{d}} f_{t}(\xi) M(d \xi), t \in \mathbb{R}^{d}\right)
$$

when the function $t \mapsto f_{t}$ is continuous with values in $L^{\alpha}\left(\mathbb{R}^{d}\right)$. We notice that the result still holds when $f_{t}$ is a vector valued function. Let us look at the following case

$$
\begin{aligned}
f_{t}(\xi) & =\left(|t-\xi|^{H-d / \alpha} q\left(\frac{t-\xi}{|t-\xi|}\right)-|\xi|^{H-d / \alpha} q\left(\frac{-\xi}{|\xi|}\right), \frac{e^{i t \xi}-1}{|\xi|^{K+d / \alpha} s(\xi /|\xi|)}\right) \\
& =\left(f_{t}^{1}(\xi), f_{t}^{2}(\xi)\right) \in \mathbb{R}^{2} .
\end{aligned}
$$

We know from Theorem 3.1

$$
\begin{aligned}
& X_{t}=\int_{\mathbb{R}^{d}} f_{t}^{1}(\xi) M(d \xi), \\
& Z_{t}=\int_{\mathbb{R}^{d}} f_{x}^{2}(\xi) M(d \xi) .
\end{aligned}
$$

It is easy to check that $\forall \eta>0$,

$$
\begin{aligned}
& \eta^{-H} f_{\eta t}^{1}(\xi)=\eta^{-d / \alpha} f_{t}^{1}\left(\frac{\xi}{\eta}\right) \\
& \eta^{+K} f_{\eta t}^{2}(\xi)=\eta^{-d / \alpha} f_{t}^{1}\left(\frac{\xi}{\eta}\right)
\end{aligned}
$$


so that (4.1) gives the inverse scaling property

$$
\forall \eta>0, \quad\left(\frac{1}{\eta^{H}} X(\eta .), \eta^{K} Z\left(\frac{\dot{\eta}}{\eta}\right)\right)=_{L a w}(X(.), Z(.)) .
$$

The I.S.I. property is now proved and the main hypothesis was (SM3). The proof of stationarity of increments are similar and we do not give any details. Using hypothesis (SM1), the translation invariance in law for $M$, we can show that the increments of the process $X$ are stationary. In order to get the same result for process $Z$ we need hypothesis (SM2). In fact as we know

$$
\mathcal{F}(f(.-t))(\xi)=\mathcal{F}(f)(\xi) e^{i t . \xi}
$$

the effect on process $Z$ is precisely the translation invariance in law of its increments.

Remark 4.1 The converse statement is valid: if $X, Z$ satisfies $\operatorname{ISI}(H, H)$ then $M$ satisfies SM1, SM2, SM3.

Remark 4.2 Let $M$ be a random measure which satisfies SM1, SM2, SM3. Let us suppose moreover that $M$ is independently scattered on $\mathbb{R}^{d}$. Then $M$ is an $\alpha$-stable measure. To prove this it is sufficient to work with rectangles $A=\prod_{j=1}^{d}\left(0, t_{j}\right)$ in $\mathbb{R}^{d}$. For every positive integer $n$, we can decompose the set $A=\prod_{j=1}^{d}\left(0, t_{j}\right)$ into $n^{d}$ nonoverlapping pieces which are images under translations of $B=\prod_{j}\left(0, \frac{t_{j}}{n}\right)$. Using hypotheses SM1, SM3 we get the identity

$$
n^{d / \alpha} T={ }_{\text {Law }} \sum_{1 \leq i \leq n^{d}} T_{i}
$$

where $T_{i}$ are independent copies of $T:=M(B)$. This leads to the very definition of $\alpha$-stable law.

\subsection{Proportionality of Laws}

We recall processes $U$ and $V$ are said with proportional laws if up to a multiplicative factor their laws are equal

$$
\exists \eta>0, \quad\left(U(t), t \in \mathbb{R}^{d}\right)={ }_{L a w}\left(\eta V(t), t \in \mathbb{R}^{d}\right) .
$$

In the case $d=1$ and Gaussian laws $(\alpha=2)$ if processes $X_{1}, X_{2}$ are both $H$-self-similar with stationary increments then they are with proportional laws. This means the F.B.M. is essentially the only one such process.

The more general $\alpha$-stable case is studied in the book of SamorodnitskyTaqqu ([13], chapter 7): the random measure is supposed to be indepently 
scattered $\alpha$-stable; the processes $Z, X$ are respectively called harmonizable and moving average fractional stable motions. It is shown in [13] that the laws of $X$ and $Z$ are not proportional if $\alpha<2$. This result can be generalized as follows.

Corollary 4.1 We suppose the conditions of Theorem 3.1 to hold, $1<\alpha \leq 2$. The processes $X, Z$ are with proportional laws if and only if $\alpha=2$.

Proof. From $(S P),\left(S P_{*}\right)$ we get

$$
L_{\alpha} L_{\alpha^{*}} X=L_{\alpha} M, \quad L_{\alpha^{*}} L_{\alpha} Z=L_{\alpha^{*}} \widehat{M} .
$$

If the laws of $X$ and $Z$ were equal up to the multiplicative factor $c$ we should get

$$
L_{\alpha} M={ }_{L a w} c L_{\alpha^{*}} \widehat{M}
$$

then $\mathcal{R}_{\alpha^{*}} L_{\alpha} M={ }_{\text {Law }} c \widehat{M}$ and as a consequence

$$
\mathcal{R}_{\alpha_{*}} L_{\alpha}=c \mathcal{F}
$$

Coming back to the definition of these operators this happens if and only if $\alpha=2$.

N.B.: In the case where the random measure $M$ is independently scattered this is the Gaussian case.

\section{Wavelet decomposition, regularity}

In this section we study regularity of paths and wavelet decomposition of processes $X, Z$ solutions of problems $\left(S P_{*}\right),(S P)$. We refer to [1] for similar results in the Gaussian case, $\alpha=2$.

Wavelet decomposition find many applications. It is the starting point for building a new family of self-similar processes in the next section. In [1] it was the key point for giving exact constants in local or uniform continuity modulus. Here we study first the Hölder continuity property of paths; various methods are available. In a first part we give a condition which insures Hölder continuous paths using Kolmogorov's criterion. The following parts are independent of the first one; we give the wavelet decomposition of both processes and then we show how it can be applied in the important case where $M$ is independently scattered to prove optimality of the given condition for path continuity. 


\subsection{Hölder continuity}

Theorem 5.1 We suppose that the hypotheses of Theorem 3.1 are fullfilled. Processes $X, Z$ are respective solutions of problems $\left(S P_{*}\right),(S P)$. If $H \alpha>d$ there exists a modification of $X$ such that almost all sample paths are Höldercontinuous of order any $\varepsilon<H \alpha-d$. The same result is true for $Z$ under condition $H \alpha^{*}>d$, with $\varepsilon<H \alpha^{*}-d$.

We use the following version of Kolmogorov's criterion, see [3], adapted to the $\mathbb{R}^{d}$ case.

Lemma 5.1 Let $\left\{\xi(x), x \in[0,1]^{d}\right\}$ a process which satisfies

$$
\mathbb{P}(|\xi(y)-\xi(x)|>g(|y-x|)) \leq f(|y-x|)
$$

where $f, g$ are non negative and non decreasing functions on $\mathbb{R}^{+}$. If

$$
\sum_{m \geq 1} 2^{m d} f\left(2^{-m}\right)<\infty
$$

and if for some $c>0$ the sequence $G_{n}=\sum_{m \geq n+1} g\left(2^{-m}\right)$ satisfies

$$
G_{n} \leq c g\left(2^{-n}\right)
$$

we can find a modification $\eta$ of $\xi$ and some constant $c^{\prime}>0$ such that with probability 1

$$
|\eta(y)-\eta(x)| \leq c^{\prime} g(|y-x|)
$$

Let $c_{1}=\max _{|u|=1} \int\left|K_{\alpha}(u, y)\right|^{\alpha} d y$, a finite constant by Lemma (2.1). After a change of scale $y=|t| y^{\prime}$ we get

$$
\int_{\mathbb{R}^{d}}\left|K_{\alpha}(t, y)\right|^{\alpha} d y \leq c_{1}|t|^{H \alpha}
$$

and using homogeneity

$$
\int_{\mathbb{R}^{d}}\left|K_{\alpha}(t, y)-K_{\alpha}(s, y)\right|^{\alpha} d y \leq c_{1}|t-s|^{H \alpha} .
$$

As the stochastic measure $M$ is of $(\alpha, p)$ type

$$
\begin{aligned}
r^{\alpha} \mathbb{P}\left(\left|X_{t}-X_{s}\right|>r\right) & =r^{\alpha} \mathbb{P}\left(\left|\tilde{K}_{\alpha} M(t)-\tilde{K}_{\alpha} M(s)\right|>r\right) \\
& \leq c_{2}\left\|K_{\alpha}(t, .)-K_{\alpha}(s, .)\right\|_{L^{\alpha}\left(\mathbb{R}^{d}\right)}^{\alpha} \\
& \leq c_{1} c_{2}|t-s|^{H \alpha}
\end{aligned}
$$

for every $r>0$. 
Let us define functions $f(\rho)=\rho^{H \alpha+\varepsilon}, g(\rho)=\rho^{\varepsilon / \alpha}$ and observe

$$
\forall n, \quad \sum_{m \geq n+1} g\left(2^{-m}\right) \leq c g\left(2^{-n}\right)
$$

with $c=\left(2^{\varepsilon / \alpha}-1\right)^{-1}$, and

$$
\sum_{m \geq 1} 2^{m d} f\left(2^{-m}\right)=\left(2^{\alpha H-d-\varepsilon}-1\right)^{-1} .
$$

Hypotheses of Lemma 5.1 are satisfied and the Theorem 5.1 is proved for process $X$. The proof for $Z$ is similar and thus omitted.

Remark 5.1 Recall $0<H<1$. If $1<\alpha \leq 2$ the condition given for regularity of trajectories of $X$ requires $d=1$. Notice that for $d>1$ we get continuous paths for restrictions to one dimensional subspaces, $(X(\tau v), \tau \in \mathbb{R})$, $v$ fixed in $\mathbb{R}^{d} \backslash\{0\}$ : such a restriction inherit ellipticity property from the process $\left(X(t), t \in \mathbb{R}^{d}\right)$. Notice also that if $H \alpha \leq d$ we can define

$$
\tilde{X}(t)=\int_{\mathbb{R}^{d}} \frac{\exp (i t \xi)-\sum_{0 \leq k \leq 1+d-\alpha H}(i t \xi)^{k} / k !}{|\xi|^{d / \alpha^{*}+H}} \widehat{s(\xi /|\xi|)(2 \pi)^{d}} \widehat{M}(d t) .
$$

which is the very process to study continuity of paths, see [1].

\subsection{Wavelet decomposition}

We denote by ||$_{\infty}$ the sup-norm on $\mathbb{R}^{d},|x|_{\infty}=\max \left|x_{i}\right|, 1 \leq i \leq d$. Let us recall well known facts about wavelet bases on $\mathbb{R}^{d}$ and define suitable functions for the following decomposition. A wavelet basis $\left(\Psi_{\lambda}\right)_{\lambda}$ on $\mathbb{R}^{d}$ is indexed by a countable set

$$
\Lambda=\left\{\lambda=(j, k, l), \quad j \in \mathbb{Z}, \quad k \in \mathbb{Z}^{d}, \quad l \in E_{d}\right\}
$$

where $E_{d}=\{0,1\}^{d} \backslash\{(0, \ldots, 0)\}$. To generate such a basis a finite family of smooth functions (mother-functions) is needed, $\mathcal{G}=\left(G_{l}\right)_{l \in E_{d}}$. It is chosen so that setting

$$
\Psi^{\lambda}(t)=G_{l}\left(2^{j} t-k\right), \quad \Psi_{\lambda}(t)=\frac{\Psi^{\lambda}(t)}{\left\|\Psi^{\lambda}\right\|_{L^{2}\left(\mathbb{R}^{d}\right)}},
$$

the family $\left(\Psi_{\lambda}\right)_{\lambda \in \Lambda}$ becomes an orthonormal basis of $L^{2}\left(\mathbb{R}^{d}\right)$. It is well known that a wavelet basis gives also an unconditional basis for most functional spaces, see [12]. Let us express this result for the case of $L^{\alpha}\left(\mathbb{R}^{d}\right)$ spaces in the following Lemma, where we use the notation

$$
\Psi_{\lambda, \alpha}(t)=\frac{\Psi^{\lambda}(t)}{\left|\Psi^{\lambda}\right|_{L^{\alpha}\left(\mathbb{R}^{d}\right)}}=c_{\alpha} 2^{j d / \alpha} G_{l}\left(2^{j} t-k\right) .
$$


Lemma 5.2 If $1<\alpha<\infty$, then $\left(\Psi_{\lambda, \alpha}\right)_{\lambda \in \Lambda}$ is an unconditional basis of $L^{\alpha}\left(\mathbb{R}^{d}\right)$.

We refer to [4] for existence of $\mathcal{G}=\left(G_{l}\right)_{l \in E_{d}}$ sharing the properties that we need in the present work: smoothness, vanishing moments up to some finite order $N$,

$$
\frac{\partial^{\mu} \widehat{G_{l}}}{\partial t^{\mu}}(0)=0 \quad \text { for }|\mu|_{\infty} \leq N
$$

and compact support. We can choose $N>m_{*}$ and then denote by $r=r(N)$ the radius of a ball centered at the origin and which contains the support of $G_{l}$ for every $l \in E_{d}$.

Let us set

$$
\theta_{l}(t)=c_{\alpha^{*}} \mathcal{R}_{\alpha^{*}}\left(\widehat{G_{l}}\right)(t)
$$

and notice that

$$
\widehat{\theta}_{l}(\xi)=\frac{c_{\alpha^{*}} \widehat{G}_{l}(\xi)}{|\xi|^{m_{*}} s(\xi /|\xi|)}
$$

makes sense since we know (5.2) is valid up to $N$. As $s$ is bounded and is greater than some $c>0$, the functions $\theta_{l}$ are smooth and rapidly decreasing at infinity. We denote by $c(\gamma)$ some positive constant such that

$$
\forall z \in \mathbb{R}^{d},\left|\theta_{l}(z)\right| \leq \frac{c(\gamma)}{1+|z|^{\gamma}} .
$$

Let us define two families of functions on $\mathbb{R}^{d}$ by setting

$$
\begin{aligned}
& \Phi_{\lambda, \alpha}(t)=\theta_{l}\left(2^{j} t+k\right)-\theta_{l}(k) \\
& \tilde{\Phi}_{\lambda, \alpha}(t)=c_{\alpha^{*}} \mathcal{R}_{\alpha}\left(G_{l}(.-k)\right)\left(2^{-j} t\right)
\end{aligned}
$$

and also a family of random variables

$$
\eta_{\lambda}=M\left(\Psi_{\lambda, \alpha}\right) .
$$

We can now get a wavelet-based decomposition for $X$ (resp. $Z$ ) the solution of problem $\left(S P_{*}\right)$ (resp. $\left.(S P)\right)$.

Theorem 5.2 We suppose that the hypotheses of Theorem 3.1 are fullfilled and that $X, Z$ are the respective solutions of problems $\left(S P_{*}\right),(S P)$. Then the following equalities

$$
\begin{aligned}
& X(t)=\sum_{\lambda \in \Lambda} 2^{-j H} \Phi_{\lambda, \alpha}(t) \eta_{\lambda} \\
& Z(t)=\sum_{\lambda \in \Lambda} 2^{j H} \tilde{\Phi}_{\lambda, \alpha}\left(2^{-j} t\right) \eta_{\lambda}
\end{aligned}
$$


hold in the distribution meaning on $\mathbb{R}^{d}$. The family $\left(\eta_{\lambda}\right)_{\lambda}$ satisfies some stationarity properties:

(i) for every fixed $(j, l)$ the sequence $\left(\eta_{\lambda}\right)_{k \in \mathbb{Z}^{d}}$ is stationary

(ii) if we set $\nu_{j}=\left(\eta_{j, k, l}\right)_{k, l}$, the sequence $\left(\nu_{j}\right)_{j \in \mathbb{Z}}$ is stationary.

Therefore the decompositions given in (5.4), (5.5) can be written as

$$
X(t)=\sum_{j \in \mathbb{Z}} 2^{-j H} X_{j}\left(2^{j} t\right), \quad Z(t)=\sum_{j \in \mathbb{Z}} 2^{j H} Z_{j}\left(2^{-j} t\right)
$$

where $\left(X_{j}(t)\right)_{j},\left(Z_{j}(t)\right)_{j}$ are stationary sequences of processes with stationary increments.

Proof. Let us first prove the following wavelet decomposition of $M$,

$$
M(d t)=\sum_{\lambda \in \Lambda} \Psi_{\lambda, \alpha^{*}}(t) M\left(\Psi_{\lambda, \alpha}\right) d t .
$$

We know that for every $\varphi \in L^{\alpha}\left(\mathbb{R}^{d}\right) \cap L^{2}\left(\mathbb{R}^{d}\right)$

$$
\varphi=\sum_{\lambda \in \Lambda}<\Psi_{\lambda}, \varphi>\Psi_{\lambda}=c_{\alpha^{*}} \sum_{\lambda \in \Lambda}<\Psi_{\lambda, \alpha^{*}}, \varphi>\Psi_{\lambda, \alpha}
$$

and as $M$ is countably additive

$$
<M, \varphi>=c_{\alpha^{*}} \sum_{\lambda \in \Lambda}<\Psi_{\lambda, \alpha^{*}}, \varphi><M, \Psi_{\lambda, \alpha}>
$$

with convergence in the $L^{p}(\Omega, \mathbb{P})$ sense. Then we get the following equalities in the distribution meaning for the solution $X$ of problem $\left(S P_{*}\right)$.

$$
\begin{aligned}
X(t) & =\mathcal{R}_{\alpha^{*}} \widehat{M}(t)=<\widehat{M}, R_{\alpha^{*}}(t, .)>=<M, \widehat{R_{\alpha^{*}}(t, .)}> \\
& =\sum_{\lambda \in \Lambda}<\Psi_{\lambda, \alpha^{*}}, \widehat{R_{\alpha^{*}}(t, .)}><M, \Psi_{\lambda, \alpha}> \\
& =c_{\alpha^{*}} \sum_{\lambda \in \Lambda} 2^{j d / \alpha^{*}} \mathcal{R}_{\alpha^{*}} \widehat{\Psi^{\lambda}}(t)<M, \Psi_{\lambda, \alpha}> \\
& =\sum_{\lambda \in \Lambda} 2^{-j H}\left(\theta_{l}\left(2^{j} t-k\right)-\theta_{l}(-k)\right)<M, \Psi_{\lambda, \alpha}> \\
& =\sum_{\lambda \in \Lambda} 2^{-j H}\left(\theta_{l}\left(2^{j} t-k\right)-\theta_{l}(-k)\right) \eta_{\lambda} .
\end{aligned}
$$

The solution $Z$ of problem $(S P)$ is given by

$$
Z(t)=\mathcal{R}_{\alpha} M(t)
$$


and using the decomposition of $M$ again we get

$$
\begin{aligned}
Z(t) & =\sum_{\lambda \in \Lambda}<\Psi_{\lambda, \alpha^{*}}, R_{\alpha}(t, .)><M, \Psi_{\lambda, \alpha}> \\
& =c_{\alpha^{*}} \sum_{\lambda \in \Lambda} 2^{j d / \alpha^{*}}<G_{l}\left(2^{j} .-k\right), R_{\alpha}(t, .)><M, \Psi_{\lambda, \alpha}> \\
& =c_{\alpha^{*}} \sum_{\lambda \in \Lambda} 2^{j d / \alpha^{*}}<G_{l}\left(2^{j} .-k\right), R_{\alpha}(t, .)>\eta_{\lambda} \\
& =c_{\alpha^{*}} \sum_{\lambda \in \Lambda} 2^{j H}<G_{l}(.-k), R_{\alpha}\left(2^{-j} t, .\right)>\eta_{\lambda} .
\end{aligned}
$$

which gives the expected formula.

The proof of stationarity properties is elementary by changing variables so we skip the proof.

\subsection{Application to path regularity}

Let us now see how the wavelet decomposition can help to get an insight into path regularity. We discuss optimality of condition $H \alpha>d$ for path continuity of $X$ in the important case where $M$ is independently scattered. We split the random wavelet sum as

$$
\begin{aligned}
\sum_{j, k, l} \eta_{j, k, l}\left(\theta_{l}\left(2^{j} t-k\right)-\theta_{l}(-k)\right)=\sum_{j \geq 0} \tilde{\rho}_{j}(t)+\sum_{j \geq 0} \tilde{\sigma}_{j}(t)+\sum_{j<0} \tilde{\tau}_{j}(t) \\
\rho_{j}(t)=\sum_{l \in E_{d},|k|_{\infty} \leq 2^{j+1}} \eta_{j, k, l} \theta_{l}\left(2^{j} t-k\right), \quad \tilde{\rho}_{j}(t)=\rho_{j}(t)-\rho_{j}(0) \\
\sigma_{j}(t)=\sum_{l \in E_{d},|k|_{\infty}>2^{j+1}} \eta_{j, k, l} \theta_{l}\left(2^{j} t-k\right), \quad \tilde{\sigma}_{j}(t)=\sigma_{j}(t)-\sigma_{j}(0) \\
\tilde{\tau}_{j}(t)=\sum_{l \in E_{d}, k \in \mathbb{Z}^{d}} \eta_{j, k, l}\left(\theta_{l}\left(2^{j} t-k\right)-\theta_{l}(-k)\right)
\end{aligned}
$$

In the following proposition we consider only $\sigma_{j}$ and $\tau_{j}$ 's. We get convergence results without assuming $H \alpha>d$ or increments of $M$ are independent. We recall $0<H<1$.

Proposition 5.1 Suppose we choose the wavelet family such that (5.3) is satisfied with $\gamma>d$. Then with probability 1, we obtain uniform convergence on bounded sets for the series $\sum_{j \geq 0} 2^{-j H} \tilde{\sigma}_{j}(t), \sum_{j<0} 2^{-j H} \tilde{\tau}_{j}(t)$. So we get continuous functions of $t$. 
Proof. We use localisation of wavelets given in (5.3) so that

$$
\begin{aligned}
\sup _{|t| \leq 1}\left|\sigma_{j}(t)\right| & \leq \sum_{q=1}^{\infty} \sum_{l \in E_{d},|k|_{\infty}=q+2^{j+1}} \sup _{|t| \leq 1}\left|\theta_{l}\left(2^{j} t-k\right)\right|\left|\eta_{j, k, l}\right| \\
& \leq \sum_{q=1}^{\infty} \frac{c(\gamma)}{1+\left|2^{j}+q\right|^{\gamma}} \sum_{l \in E_{d},|k|_{\infty}=q+2^{j+1}}\left|\eta_{j, k, l}\right| .
\end{aligned}
$$

We recall $\eta_{\lambda}=M\left(\Psi_{\lambda, \alpha}\right)$ with functions $\Psi_{\lambda, \alpha}$ normalized in $L^{\alpha}\left(\mathbb{R}^{d}\right)$ and (3.1) holds; then we get

$$
\begin{aligned}
& \mathbb{E}\left(\sup _{|t| \leq 1}\left|\sigma_{j}(t)\right|\right) \leq c_{1} \sum_{q=1}^{\infty} \frac{1}{1+\left|2^{j}+q\right|^{\gamma}} \sum_{l \in E_{d},|k|_{\infty}=q+2^{j+1}}\left|\Psi_{j, k, l, \alpha}\right|_{L^{\alpha}} \\
& \leq c_{2} \sum_{q=1}^{\infty} \frac{1}{1+\left|2^{j}+q\right|^{\gamma}}\left(2^{d}-1\right)\left(q+2^{j+1}\right)^{d-1} \leq c_{3} \sum_{q=1}^{\infty} \frac{1}{q^{1+\gamma-d}}=c_{4} .
\end{aligned}
$$

Let us turn to $j<0$. We rely now upon smoothness of $\theta$ functions. We know that for $j<0$ and $|t| \leq 1$

$$
\left|\theta_{l}\left(2^{j} t-k\right)-\theta_{l}(-k)\right| \leq 2^{j} \max _{1 \leq i \leq d,|z-k| \leq 1}\left|\frac{\partial \theta_{l}}{\partial z_{i}}(z)\right|
$$

and therefore

$$
\mathbb{E}\left(\sup _{|t| \leq 1}\left|\tilde{\tau}_{j}(t)\right|\right) \leq 2^{j} c_{5} \sum_{q=1}^{\infty} \frac{1}{1+q^{\gamma}} \sum_{|k|_{\infty}=q, l \in E_{d}} \mathbb{E}\left|\eta_{j, k, l}\right| \leq c_{6} 2^{j}
$$

Using Markov inequality we get

$$
\mathbb{P}\left(\sup _{|t| \leq 1}\left|\sigma_{j}(t)\right| \geq 2^{j H / 2}\right) \leq c_{4} 2^{-j H / 2}, \mathbb{P}\left(\sup _{|t| \leq 1}\left|\tilde{\tau}_{j}(t)\right| \geq 2^{j(1+H) / 2}\right) \leq c_{6} 2^{j(1-H) / 2}
$$

and from the Borel-Cantelli Lemma, with probability 1

$$
\begin{gathered}
\sup _{|t| \leq 1} 2^{-j H}\left|\sigma_{j}(t)\right|<2^{-j H / 2}, \quad \text { if } j \text { large enough } \\
\sup _{|t| \leq 1} 2^{-j H}\left|\tilde{\tau}_{j}(t)\right| \leq 2^{j(1-H) / 2}, \quad \text { if }-j \text { large enough. }
\end{gathered}
$$

The uniform convergence of $\sum_{j \geq 0} 2^{-j H} \sigma_{j}$ and of $\sum_{j<0} 2^{-j H} \tilde{\tau}_{j}$ on $T=$ $|t| \leq 1$ follows. The same is true for $\sum_{j>0} 2^{-j H} \tilde{\sigma}_{j}$ and it extends to every bounded $T$ in $\mathbb{R}^{d}$. 
It remains to study $\sum_{j>0} 2^{-j H} \tilde{\rho}_{j}(t)$. From now on we suppose $M$ is with independent increments.

\section{Case $H \alpha>d$}

We partition the set $\mathbb{Z}^{d}$ into $(2 i+1)^{d}$ pieces $P_{h}=h+i \mathbb{Z}^{d},|h|_{\infty} \leq i$ where the integer $i$ is chosen such that $2 i+1 \geq r(N)$; with this choice the supports of $\Psi_{j, k, l, \alpha}, \Psi_{j, k^{\prime}, l, \alpha}$ are disjoint for distinct $k, k^{\prime}$ in the same $P_{h}$. Let us work with $h$ and $l$ fixed and study uniform convergence of $\sum_{j>0} 2^{-j H} \rho_{j ; h, l}(t)$, where

$$
\rho_{j ; h, l}(t)=\sum_{k \in Q(j, h)} \theta_{l}\left(2^{j} t-k\right) \eta_{j, k, l}, \quad \text { and } Q(j, h)=\left\{k \in P_{h},|k|_{\infty} \leq 2^{j+1}\right\} .
$$

We know here that $M$ is independently scattered. It is then an $\alpha$-stable stochastic measure, see Remark 4.2. Using the tail behaviour of $l^{1}$ norm for a stable vector, see [13, p. 16 and 197], and disjoint supports of $\Psi_{j, k, l, \alpha}$ we find

$$
\mathbb{P}\left(\sum_{Q(j, h)}\left|M\left(\Psi_{j, k, l, \alpha}\right)\right|>r\right) \sim K(\alpha) r^{-\alpha} \operatorname{card}(Q(j, h))
$$

when $r \rightarrow+\infty$, with $K(\alpha)=(1-\alpha) / \cos (\alpha / 2) \Gamma(2-\alpha)$. As $\theta_{l}$ are bounded functions on $\mathbb{R}^{d}$ we get

$$
\mathbb{P}\left(\sup _{|t| \leq 1}\left|2^{-j H} \rho_{j ; h, l}(t)\right| \geq 2^{-j \varepsilon}\right) \leq c_{1} 2^{-j \alpha(H-\varepsilon)} 2^{(j+1) d} .
$$

By choosing $0<\varepsilon<H-d / \alpha$ and we deduce from Borel-Cantelli Lemma that almost surely for $j$ large enough,

$$
\sup _{|t| \leq 1}\left|2^{-j H} \rho_{j ; i, l}(t)\right|<\infty .
$$

Uniform convergence and continuity of the sum are easy consequences. We have proved that almost all paths of $X$ are continuous in the present case.

Case $H \alpha<d$

Reminding Prop. 5.1 the only sum to pay attention with is $\sum_{j \geq 0} 2^{-j H} \rho_{j}(t)$. As $\theta$ is rapidly decreasing we can heuristically replace $\rho_{j}(t)$ by

$$
\overline{\rho_{j}}(t)=\sum_{\left|k-2^{j} t\right| \leq R, l \in E_{d}} \theta\left(2^{j} t-k\right) \eta_{j, k, l}
$$

with $R$ some positive radius. Let us consider a bounded domain like $D=$ $(1,2)^{d}$ and the discrete set $V_{j}=(1,2)^{d} \cap R 2^{-j} \mathbb{Z}^{d}$. We get an independent sequence $\left(\overline{\rho_{j}}(t)\right)_{t \in V_{j}}$ such that, for all $t$ in $V_{j}$,

$$
\mathbb{P}\left(\left|\overline{\rho_{j}}(t)\right|>r\right) \geq c_{1} r^{-\alpha}\left(\left|\sum_{\left|k-2^{j} t\right| \leq R, l} \theta\left(2^{j} t-k\right) \Psi_{\lambda, \alpha}(.)\right|_{L^{\alpha}}\right)^{\alpha} \geq c_{2} r^{-\alpha} .
$$


As $M$ is independently scattered,

$\mathbb{P}\left(\max _{t \in V_{j}} 2^{-j H}\left|\tilde{\rho}_{j}(t)\right| \leq j\right)=\prod_{t \in V_{j}} 1-\mathbb{P}\left(\left|\overline{\rho_{j}}(t)\right|>j 2^{j H}\right) \leq\left(1-c_{2}\left(j 2^{j H}\right)^{-\alpha}\right)^{\left(2^{j} / R\right)^{d}}$.

The sum $\sum_{j>0}\left(1-c_{2}\left(j 2^{j H}\right)^{-\alpha}\right)^{\left(2^{j} / R\right)^{d}}$ being finite we know from BorelCantelli Lemma

$$
2^{-j H} \max _{t \in V_{j}}\left|\overline{\rho_{j}}(t)\right| \geq j
$$

almost surely for $j$ large. We can conclude that with probability 1 the paths of process $X$ are unbounded on $(1,2)^{d}$. The same is true on every domain in $\mathbb{R}^{d}$.

\section{Examples}

In this paragraph we shall give examples of elliptic self-similar processes with stationary increments.

Example 1. $\alpha=2,\left(=\alpha_{*}\right)$ and $M$ is the Gaussian white noise. In this situation $X$ and $Z$ are essentially the same process: their laws are equal up to a multiplicative factor.

Example 2. $1<\alpha<2$ and $M$ is the symmetric $\alpha$-stable measure. In this case, $X$ and $Z$ are fractional stable processes which are thoroughly studied in [13].

Example 3. In the third example the law of the process will be non stable. We follow ideas of [2] where a model of intermittency is proposed. It rests upon the following remark: the set $\Lambda$ which indices the wavelet basis can be written as

$$
\Lambda=T_{2^{d}} \times E_{d}
$$

if we denote by $T_{q}$ the regular $q$-tree (from each vertex $(q+1)$ edges are drawn). We restrict ourselves to the case $d=1$ in which case $E_{1}=\{1\}$ and $\Lambda$ reduces to $T_{2}$. Using the 2-adic tree $T_{2}$ we can encode the dyadic cells of $\mathbb{R}$, in such a way that the significative values of $\psi_{\lambda}$ are taken on the cell $C_{\lambda}$. Now let $X$ an elliptic symmetric self-similar process with stationary increments. The decomposition given in (5.4) can be now written as

$$
X(x)=\sum_{\lambda \in T_{2}} 2^{-j H} \Phi_{\lambda, \alpha}(x) \eta_{\lambda} .
$$

In order to define intermittency in [2] the summation was restricted to a subtree of $T_{2}$. But we loose the self similarity property in doing this. Therefore we restrict the summation to a forest $\mathcal{F}$ build from a family of subtrees of $T_{2}$. We shall choose a percolation forest. 
Let $\mathcal{B}_{p}$ be the Bernoulli law with parameter $p, p \in(1 / 2,1]$. Let $\left(B_{\lambda}\right)_{\lambda \in T_{2}}$ an i.i.d. family of r.v. with law $\mathcal{B}_{p}$. We say that an edge $\lambda \in T_{2}$ is open if $B_{\lambda}=1$ and closed otherwise. An infinite percolation cluster is a connected infinite set of open edges of $T_{2}$. Let $A$ be such a percolation set and $r(A)$ the vertex in $A$ which is the closest to the root $*$. Then $(A, r(A))$ is a subtree of $T_{2}$, which is called a percolation subtree. We define the forest $\mathcal{F}_{p}$ as the union of all percolation subtrees.

Every permutation on $T_{2}$ acts on $\left(B_{\lambda}\right)_{\lambda \in T_{2}}$ and induces a permutation of the forest, denoted by $\sigma \mathcal{F}_{p}$. Then we know from [8]

\section{Lemma 6.1}

(i) for any permutation $\sigma$ of $T_{2}$,

$$
\operatorname{Law}\left(\mathcal{F}_{p}\right)=\operatorname{Law}\left(\sigma \mathcal{F}_{p}\right)
$$

(ii) If $p>1 / 2$, with probability 1 we have

$$
\mathcal{F}_{p} \neq \emptyset
$$

and $\mathcal{F}_{p}$ contains infinitely many percolation subtrees.

Let $M$ be a symmetric $\alpha$-stable stochastic measure on $\mathcal{R}^{d}$, and let us define the stochastic process $\left\{Y_{p}(x), x\right\}$ for $p \in(1 / 2,1]$ as follows

$$
Y_{p}(x)=\sum_{\lambda \in \mathcal{F}_{p}} 2^{-j H} \Phi_{\lambda, \alpha}(x) \eta_{\lambda}
$$

Theorem 6.1 If we suppose $M$ and $\left(B_{\lambda}\right)_{\lambda \in T_{2}}$ stochastically independent and $p>1 / 2$ the process $Y_{p}$ is symmetric $H$-self-similar and with stationary increments.

Proof. Let us define a stochastic measure $M_{p}$ by

$$
M_{p}(d x)=\sum_{\lambda \in \mathcal{F}_{p}} \Psi_{\lambda, \alpha^{*}}(x) \eta_{\lambda} d x
$$

Then it can be checked that the conditions of Theorem 3.3 are satisfied and consequently that $Y_{p}$ is the solution of

$$
L_{\alpha^{*}} Y_{p}=M_{p}, \quad Y_{p}(0)=0 .
$$

We give now the proofs of the properties (SM1), (SM2), (SM3) for $M_{p}$, and we use freely the formal notation

$$
<T, \varphi>=\int_{\mathbb{R}^{d}} \varphi(x) T^{\prime}(x) d x
$$

when $T$ is a distribution, possibly random. 
Proof of scaling property for $M_{p}$.

Let us change the variable $x \in \mathbb{R}^{d}$ in $\mu x$, for some $\mu>0$. We get

$$
\mu^{d} M_{p}^{\prime}(\mu x)=\sum_{\lambda \in \mathcal{F}_{p}} \mu^{d / \alpha^{*}} \Psi_{\lambda, \alpha^{*}}(\mu x) M\left(\mu^{d / \alpha} \Psi_{\lambda, \alpha}\right) .
$$

But $M\left(\mu^{d / \alpha} \Psi_{\lambda, \alpha}\right)=\mu^{d}<M^{\prime}(\mu),. \mu^{d / \alpha} \Psi_{\lambda, \alpha}(\mu)>$. and we get the following equality in law

$$
M\left(\mu^{d / \alpha} \Psi_{\lambda, \alpha}\right)={ }_{L a w} \mu^{d / \alpha}<M^{\prime}(), \mu^{d / \alpha} \Psi_{\lambda, \alpha}(\mu .)>.
$$

So if we define new functions on $\mathbb{R}^{d}$ by

$$
\Psi_{\lambda, \alpha}^{[\mu]}(x)=\mu^{d / \alpha} \Psi_{\lambda, \alpha}(\mu x), \quad \Psi_{\lambda, \alpha^{*}}^{[\mu]}(x)=\mu^{d / \alpha^{*}} \Psi_{\lambda, \alpha^{*}}(\mu x)
$$

we get new unconditional bases of respectively $L^{\alpha}\left(\mathbb{R}^{d}\right)$ and $L^{\alpha^{*}}\left(\mathbb{R}^{d}\right)$. And we can write

$$
\mu^{d} M_{p}^{\prime}(\mu x)={ }_{L a w} \sum_{\lambda \in \mathcal{F}_{p}} \mu^{d / \alpha} \Psi_{\lambda, \alpha^{*}}^{[\mu]}(x) M\left(\Psi_{\lambda, \alpha}^{[\mu]}(x)\right) .
$$

If we denote by $\mathcal{F}_{p}^{[\mu]}$ the percolation forest associated with $T_{2}$ encoding the dyadic cells when unit length is $\mu$, we deduce from Lemma (6.1) that

$$
\operatorname{Law}\left(\mathcal{F}_{p}^{[\mu]}\right)=\operatorname{Law}\left(\mathcal{F}_{p}\right)
$$

thus the following equality in law

$$
\sum_{\lambda \in \mathcal{F}_{p}} \Psi_{\lambda, \alpha^{*}}^{[\mu]}(x) M\left(\Psi_{\lambda, \alpha}^{[\mu]}(x)\right)={ }_{L a w} \sum_{\lambda \in \mathcal{F}_{p}^{[\mu]}} \mu^{d / \alpha} \Psi_{\lambda, \alpha^{*}}^{[\mu]}(x) M\left(\Psi_{\lambda, \alpha}\right) .
$$

Finally

$$
\mu^{d} M_{p}^{\prime}(\mu x)={ }_{L a w} \mu^{d / \alpha} M_{p}^{\prime}(x)
$$

which is the scaling property we looked for.

Proof of stationarity property for $M_{p}$.

Thanks to the previous proof we have only to show that

$$
M_{p}^{\prime}(x-1)={ }_{\text {Law }} M_{p}^{\prime}(x)
$$


But this is a consequence of the following equalities

$$
\begin{aligned}
M_{p}^{\prime}(x-1) & =\sum_{\lambda \in \mathcal{F}_{p}} \Psi_{\lambda, \alpha^{*}}(x-1) M\left(\Psi_{\lambda, \alpha}\right) \\
& =\sum_{\lambda \in \mathcal{F}_{p}} \Psi_{\lambda, \alpha^{*}}(x-1)<M^{\prime}(.-1), \Psi_{\lambda, \alpha}(.-1)> \\
& \left.={ }_{L a w} \sum_{\lambda \in \mathcal{F}_{p}} \Psi_{\lambda, \alpha^{*}}(x-1)<M^{\prime}(.), \Psi_{\lambda+1, \alpha}\right)> \\
& \left.=\sum_{\lambda \in \mathcal{F}_{p}} \Psi_{\lambda+1, \alpha^{*}}(x)<M^{\prime}(.), \Psi_{\lambda+1, \alpha}\right)> \\
& \left.={ }_{\text {Law }} \sum_{\lambda \in \mathcal{F}_{p}^{+1}} \Psi_{\lambda, \alpha^{*}}(x)<M^{\prime}(.), \Psi_{\lambda, \alpha}\right)>
\end{aligned}
$$

where $\mathcal{F}_{p}^{+1}=\left\{\lambda+1, \quad \lambda \in \mathcal{F}_{p}\right\}$ and of Lemma (6.1).

Proof of unitarity property for $M_{p}$.

This property SM2 amounts to show SM1 for $\widehat{M}$, which is proved just as above.

\section{References}

[1] Benassi, A., Jaffard, S., Roux, D.: Elliptic Gaussian random processes. Rev. Mat. Iberoamericana 13 (1997), no. 1, 19-90.

[2] Benassi, A., Cohen, S., Deguy, S., Istas, J.: Self-similarity and intermittency. In Wavelets and Signal Processing. Birkhäuser, 2003.

[3] Cramer, H., Leadbetter, M. R.: Stationary and Related Stochastic Processes. Sample function properties and their applications. John Wiley and Sons, Inc., New York-London-Sydney, 1967.

[4] Daubechies, I.: Ten Lectures on Wavelets. Regional Conference Series in Applied Mathematics 61. Society for Industrial and Applied Mathematics, Philadelphia, PA, 1992.

[5] Dobrushin, R. L.: Gaussian and their subordinated self-similar random generalised fields. Ann. Probab. 7 (1979), no. 1, 1-28.

[6] Gelfand, I. M., Vilenkin, N. Y.: Generalised functions, volume 4. Moscow, 1961.

[7] JAFFARD, S.: Old friends revisited: the multifractal nature of some classical functions. J. Fourier Anal. Appl. 3 (1997), no. 1, 1-22.

[8] Lyons, R.: Random walk and percolation on a tree. Ann. Probab. 18 (1990), 937-958.

[9] Mandelbrot, B. B.: Fractals: form, chance, and dimension.. W. H. Freeman and Co., San Francisco, Calif., 1977. 
[10] Maejima, M.: On a class of self-similar processes. Z. Wahrsch. Verw. Gebiete 62 (1983), 235-245.

[11] Mandelbrot, B. B., Van Ness, J. W.: Fractional Brownian motions, fractional noises and applications. SIAM Rev. 1 (1968), 422-437.

[12] Meyer, Y.: Ondelettes et opérateurs I. Ondelettes. Actualités Mathématiques. Hermann, Paris, 1990.

[13] Samorodnitsky, G., Taqqu, M. S.: Stable non Gaussian random processes: stochastic models with infinite variance. Chapman-Hall, New York, 1994.

[14] Schwartz, L.: Théorie des distributions. Hermann, Paris, 1966.

[15] TAqqu, M.S., Wolpert, R. L.: Infinite variance self-similar processes subordinated to a Poisson measure. Z. Wahrsch. Verw. Gebiete 62 (1983), $53-72$.

[16] Titchmarsh, E. C.: Introduction to the theory of Fourier Integrals. Oxford University Press, 1937.

Recibido: 19 de octubre de 2000

Revisado: 7 de abril de 2002

Albert Benassi

LaMP, CNRS UPRESA 6016

Université Blaise Pascal

(Clermont-Ferrand 2), 63177 Aubière Cedex (France)

benassi@opgc.univ-bpclermont.fr

Daniel Roux

LMA, CNRS UMR 6620

Université Blaise Pascal

(Clermont-Ferrand 2), 63177 Aubière Cedex (France)

Daniel.Roux@math.univ-bpclermont.fr 